\title{
Archéopages
}

Archéopages

Archéologie et société

\section{Étude des matériaux de construction en terre crue des sites antiques de Rirha (Maroc)}

Cécilia Cammas et Jean-Claude Roux

\section{(2) OpenEdition}

1 Journals

Édition électronique

URL : https://journals.openedition.org/archeopages/1221

DOI : 10.4000/archeopages. 1221

ISSN : 2269-9872

Éditeur

INRAP - Institut national de recherches archéologiques préventives

Édition imprimée

Date de publication : 1 mars 2016

Pagination : 68-69

ISSN : $1622-8545$

\section{Référence électronique}

Cécilia Cammas et Jean-Claude Roux, «Étude des matériaux de construction en terre crue des sites antiques de Rirha (Maroc) », Archéopages [En ligne], 42 | 04-07/2015, mis en ligne le 01 juillet 2017, consulté le 03 juin 2021. URL : http://journals.openedition.org/archeopages/1221 ; DOI : https:// doi.org/10.4000/archeopages. 1221

Ce document a été généré automatiquement le 3 juin 2021.

(c) Inrap 


\title{
Étude des matériaux de construction en terre crue des sites antiques de Rirha (Maroc)
}

\author{
Cécilia Cammas et Jean-Claude Roux
}

1 Les sites successifs de Rirha (Sidi Slimane, Maroc) ${ }^{1}$ sont installés dans la riche plaine agricole du Gharb. La zone archéologique, en domaine alluvial, est actuellement enserrée dans un méandre récent du Beht. La fouille porte sur un tell dont l'occupation débute à l'âge du Fer, et qui est vraisemblablement agrandi durant l'époque romaine, puis réoccupé à l'époque médiévale entre le $\mathrm{XII}^{\mathrm{e}}$ et le $\mathrm{XIV}^{\mathrm{e}}$ siècle. Pour la période maurétanienne, la terre crue - plus particulièrement la brique - est exclusivement privilégiée pour la construction. Ce site offrait l'occasion d'étudier ce matériau, et un programme de recherche interdisciplinaire sur l'emploi de la terre crue en architecture $^{2}$ (archéologique et micromorphologique) intégré au programme de fouille a été défini. Ce projet visait, pour la partie de terrain (J.-C. Roux), à identifier les élévations en terre crue et la mise en œuvre des briques dans les bâtiments (sousbassements, élévations, chaînages). Une typologie macroscopique des briques, fondée sur le format, les dimensions, la texture, la couleur des briques et les inclusions a été élaborée, puis chaque type de brique a été échantillonné pour l'analyse micromorphologique, ainsi que les joints et les enduits. Des prélèvements comprenant deux briques accolées, ou deux briques superposées, ont été réalisés afin de caractériser briques et joints. L'analyse des briques, coupées selon différents plans, avait pour but d'identifier des caractères liés au tassement dans un moule, ou encore les aménagements du sol au moment du moulage ou du séchage (Roux et Cammas, sous presse). L'objectif de l'analyse micromorphologique était de compléter la caractérisation de la terre utilisée, ainsi que les traces de façonnage, difficilement perceptibles à l'œil nu. La démarche présentée ici peut être transposée pour l'étude de tout lot d'éléments modulaires en terre crue.

2 Pour l'époque maurétanienne, dix briques ont été analysées. Les résultats montrent que les sédiments ont une texture assez variée, et qu'ils proviennent de différents dépôts 
alluviaux à composante éolienne plus ou moins marquée. Sur le terrain et à l'échelle microscopique, des inclusions anthropiques diverses ont été observées: des charbons, des fragments d'os à différents degrés de combustion, des matériaux de construction (matériaux malaxés chauffés ou non), des granules de céramique. Leur faible proportion suggère que leur présence est aléatoire ; néanmoins, ils indiquent que les premières étapes de la chaîne opératoire (prélèvement, malaxage) se déroulaient à proximité des zones anthropisées. La dimension et l'abondance des résidus végétaux attestent, quant à elles, d'un ajout volontaire, et les analyses archéobotaniques menées par Emmanuelle Bonnaire indiquent que des déchets végétaux divers, issus de différentes étapes de traitement des végétaux, étaient incorporés aux briques (Bonnaire, sous presse). L'abondance des agrégats résiduels est inversement proportionnelle au degré de malaxage, ici la variation de la composition d'une brique à l'autre montre que les éléments d'une même élévation peuvent présenter des degrés de malaxage variés. L'aplatissement des faces supérieures ou inférieures de certaines briques témoigne du tassement de la terre dans les moules, et les empreintes végétales résultent de l'utilisation de bouchons de paille pour tasser la terre, ou de lits de végétaux épandus au sol au moment du moulage. En lame mince, l'identification d'agrégats roulés par le piétinement entre les assises suggère que le bâtisseur devait se placer sur le mur au moment de la construction. Les résultats de ces travaux montrent l'absence de standardisation en ce qui concerne la terre utilisée et le degré de malaxage. La juxtaposition de briques différentes au sein d'une même élévation [ill. 1] suggère des normes souples mais précises pour la confection des briques, plutôt que des savoir-faire différents. Les mises en œuvre, sur un, deux ou trois rangs de briques disposées en parpaing, en boutisse ou en panneresse, se composent d'éléments divers selon l'épaisseur du mur; des formats se distinguent particulièrement: $34-35 \mathrm{~cm} \mathrm{x}$ $55-57 \mathrm{~cm}$ et $36-38 \mathrm{~cm} \times 54-58 \mathrm{~cm}$, avec un module plus constant de $34-36 \mathrm{~cm} \times 49-52 \mathrm{~cm}$. La réutilisation de briques crues d'un état à l'autre a pu être mise en évidence. 
1. Lame mince dans trois briques crues maurétaniennes (scan à gauche). Sous le microscope, la brique 1 présente des fantômes végétaux qui témoignent de la présence d'un dégraissant végétal (photo en haut à gauche). Dans la brique 2 , des agrégats résiduels marquent un malaxage modéré (photo en haut à droite). La brique 3 présente des graines carbonisées (photo en bas à gauche).

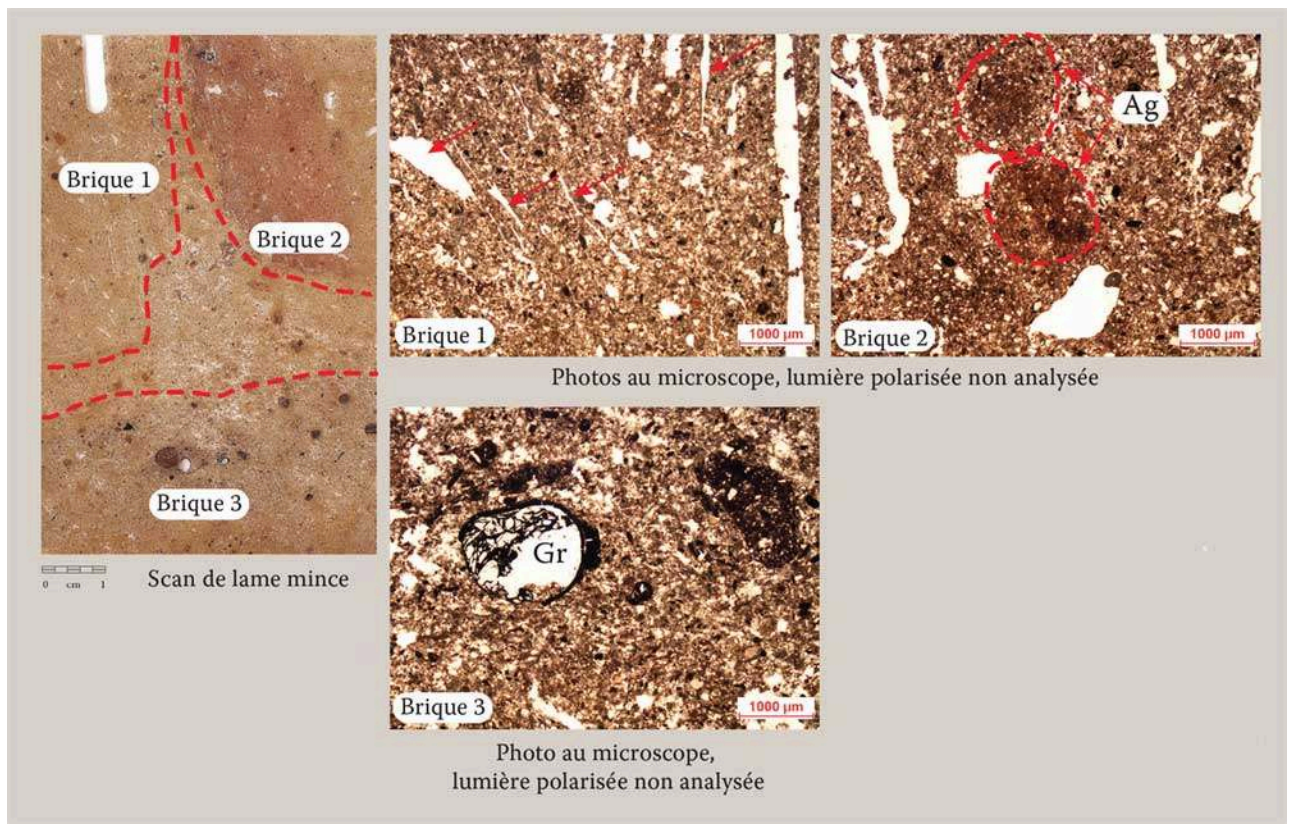

(c) C. Cammas, Inrap

Dans la domus en cours de fouille, la découverte d'un mur en terre massive du $\mathrm{II}^{\mathrm{e}}$ siècle, dont le mode de construction était énigmatique, a été l'occasion de tester les clés de détermination élaborées à l'échelle microscopique entre la bauge et le pisé. Les résultats de l'analyse montrent que les sédiments, à dominante limono-argileuse, présentent d'abondantes inclusions anthropiques grossières, comme pour les briques maurétaniennes, mais ici des agrégats de chaux sont également présents. Les sédiments ne sont pas malaxés, et la microstructure indique que les agrégats ont été entassés dans un état moyennement humide, ce qui permet le tassement mécanique. Ainsi, les caractères micromorphologiques suggéraient qu'il s'agissait d'un mur en pisé [ill. 2]. La reprise des fouilles, l'année suivante, a permis de trouver les trous de clés supportant le coffrage, ce qui confirmait ainsi l'utilisation de la technique du pisé. à travers ces exemples, les apports de la micromorphologie à la connaissance de la chaîne opératoire se dessinent. Plus particulièrement, le lieu de provenance des terres, les ajouts au moment de la préparation du matériau en terre à bâtir (addition d'eau, de végétaux) et son degré de plasticité au moment de la confection (briques) et à la pose (pisé, liants, enduits...) sont des informations originales que la micromorphologie peut apporter. 
2. Lame mince au cœur du mur en terre massive (scan à gauche). Sous le microscope, le réseau de fissures résulte de la déformation et de l'accommodation des agrégats entre eux (photo en haut à gauche et en bas à droite). L'interface entre les lits est marquée par des fissures subhorizontales, l'orientation des fissures marque une surface de tassement des sédiments (photo en bas à gauche).

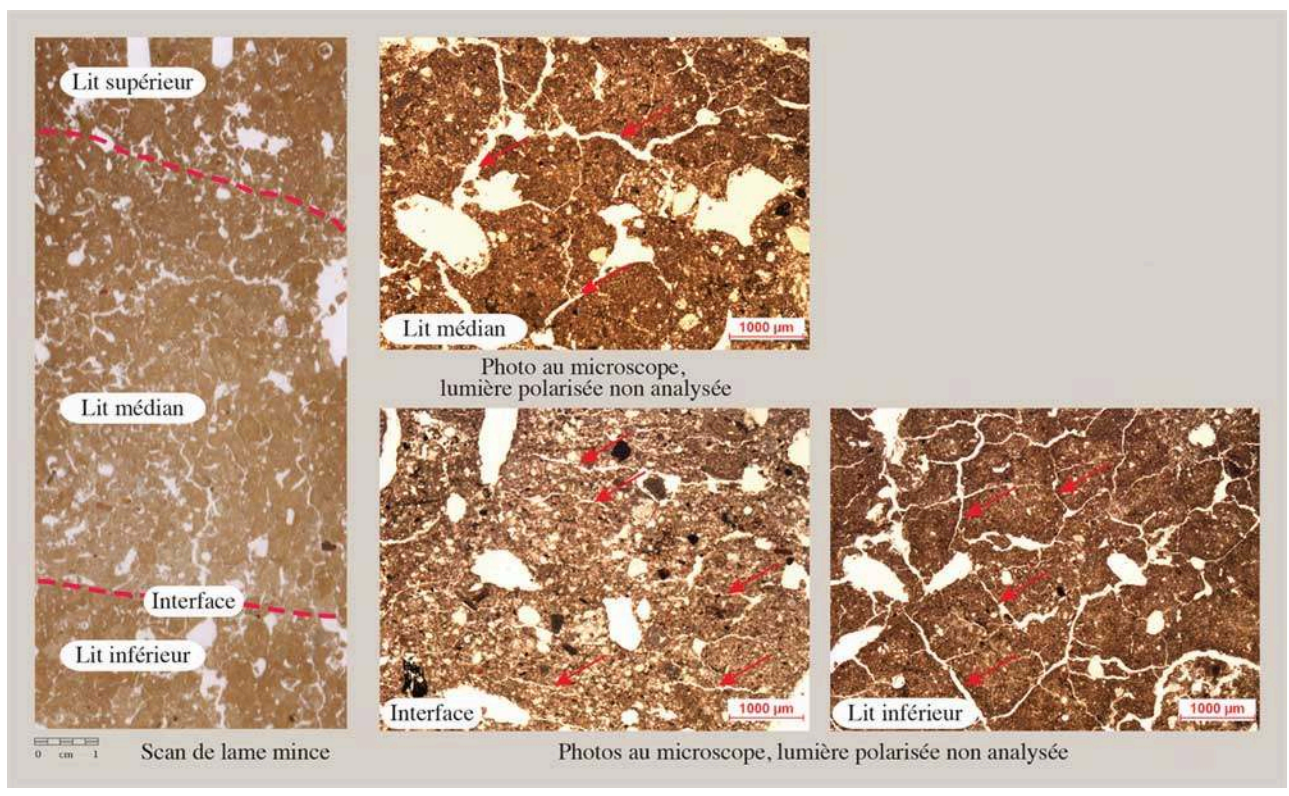

(c) C. Cammas, Inrap

\section{BIBLIOGRAPHIE}

BONNAIRE E., sous presse, « Étude archéobotanique », in CALLEGARIN L., KBIRI ALAOUI M. et ICHKHAKH A. (dir.), Le site de Rirha (Sidi Slimane, Maroc). Les occupations antique et médiévale, Madrid, Mélanges de la Casa de Velazquez.

ROUX J.-C., CAMMAS C., sous presse, « L'architecture en terre crue maurétanienne », in CALLEGARIN L., KBIRI ALAOUI M. ЕT ICHKHAKH A. (dir.), Le site de Rirha (Sidi Slimane, Maroc). Les occupations antique et médiévale, Madrid, Mélanges de la Casa de Velazquez.

\section{NOTES}

1. Fouilles dirigées par L. Callegarin jusqu'en 2013, puis par C.-A. de Chazelles.

2. Mené par les auteurs de cet article. 


\section{AUTEURS}

\section{CÉCILIA CAMMAS}

Inrap, UMR 5140, « Archéologie des sociétés méditerranéennes »

JEAN-CLAUDE ROUX

ministère de la Culture, UMR 5140, « Archéologie des sociétés méditerranéennes » 